\title{
\begin{tabular}{l|l} 
Mitraries & DSpace@MIT
\end{tabular}
}

\author{
MIT Open Access Articles
}

\section{Ultratrace Detection of Toxic Chemicals: Triggered Disassembly of Supramolecular Nanotube Wrappers}

The MIT Faculty has made this article openly available. Please share how this access benefits you. Your story matters.

Citation: Ishihara, Shinsuke et al. "Ultratrace Detection of Toxic Chemicals: Triggered Disassembly of Supramolecular Nanotube Wrappers." Journal of the American Chemical Society 138, 26 (June 2016): 8221-8227 (c) 2016 American Chemical Society

As Published: http://dx.doi.org/10.1021/jacs.6b03869

Publisher: American Chemical Society (ACS)

Persistent URL: http://hdl.handle.net/1721.1/115104

Version: Author's final manuscript: final author's manuscript post peer review, without publisher's formatting or copy editing

Terms of Use: Article is made available in accordance with the publisher's policy and may be subject to US copyright law. Please refer to the publisher's site for terms of use. 


\section{INTRODUCTION}

Low cost and portable chemical sensors facilitate personal monitoring and sharing information of hazardous chemical substances (e.g., toxic gases, explosives, and carcinogens), which are of increasing interest for security, occupational safety, and health. ${ }^{1,2}$ Chemiresistive sensors are attractive devices to realize distributed low cost sensors and operate on a simple variation of electrical conductivity of sensing materials in response to analytes of interest. ${ }^{3}$ The direct electrical nature of the transduction in these devices is ideal for integration into omnipresent electronic devices. Various types of conductive materials (e.g., carbon, ${ }^{3}$ metal oxides, ${ }^{3}$ and metal-organic-frameworks ${ }^{4}$ ) can be employed in chemiresistive sensors. We have been particularly interested in semiconductive single walled carbon nanotubes (SWCNT) as a result of their high chemical and thermal stability, relatively small responses to humidity, and compatibility with solvent-mediated processes. ${ }^{5-8}$ SWCNTs do not possess intrinsic selectivity for specific target analyte molecules and hence a major element of creating useful sensors from these materials involves functionalization with chemical units that enable selective responses to molecules or classes of reactive compounds. Chemical selectivity is commonly imparted upon SWCNTs by covalent attachment of selectors or receptors, resulting in robust chemiresistor stability. However, reactive functionalization of graphene surfaces disrupts the $\pi$-system, thereby negatively impacting sensitivity by limiting the dynamic range of the resisitivity. Conversely, non-covalent functionalization methods are less perturbative to the $\pi$-electronic structure of SWCNTs. ${ }^{9}$ Modification of SWCNTs ideally enhance a chemiresistive responses (defined as $\left(\mathrm{G}_{1}-\mathrm{G}_{0}\right) / \mathrm{G}_{0} \times 100(\%)$, where $\mathrm{G}_{0}$ and $\mathrm{G}_{1}$ are initial and measured conductance) to target analytes relative to other chemicals in the surroundings. A central goal is to develop methods that increase chemiresistive responses to target analytes relative background noise, and this need is particularly important when the target analytes are toxic at trace (parts per million or lower) concentrations. ${ }^{10}$
The cumulative exposure of toxic chemicals at trace concentrations is often of interest, and chemical dosimeters offer an important means for quantifying these events. We have previously created physical dosimeters from multi-walled carbon nanotubes wrapped with insulating poly(olefin sulfone)s displaying large increases in conductivity (ca. 10,000\%) by radiation-induced degradation of the resistive polymer wrapper to create direct MWCNTMWCNT contacts. ${ }^{11}$ In this case, evaporation of degradation products of poly(olefin sulfone)s (as $\mathrm{SO}_{2}$ and olefin) is critical for promoting direct MWCNT-MWCNT contacts. Although a limited number of synthetic polymers produce volatile degradation products, this concept has the potential for general utility to create chemiresistive dosimeters. As a result, we are engaged in creating supramolecular polymers ${ }^{12-15}$ that wrap and isolate CNTs and disassemble in response to chemical stimuli.

Herein we report a chemical dosimeter comprised of SWCNTs wrapped with a metallo-supramolecular polymer (MSP) that displays large, time- and concentration-integrated chemiresistive responses via MSP disassembly triggered by analyte molecules. We have specifically targeted strong electrophilic analytes such as diethylchlorophosphate (DECP, a reactive simulant of nerve agents) and thionyl chloride ( $\mathrm{SOCl}_{2}$, a reactive simulant of pulmonary agents). Critical to our design of chemical dosimeters is the creation of a system wherein the cooperative interactions of MSP are capable of effectively dispersing SWCNTs and maintaining them in an insulated, highly resistive state, while the MSP monomers alone interact sufficiently weakly with SWCNTs such that they are ineffective at creating a dispersion. With these conditions established, we expect that triggered disassembly of the MSP generates a conductive network with strong SWCNT-SWCNT interactions. The large and irreversible chemiresistive response associated with this process can be easily detected and we illustrate this feature using a commercial smartphone and modified near-field communication (NFC) tags to create a wireless system to detect harmful electrophiles. 
a

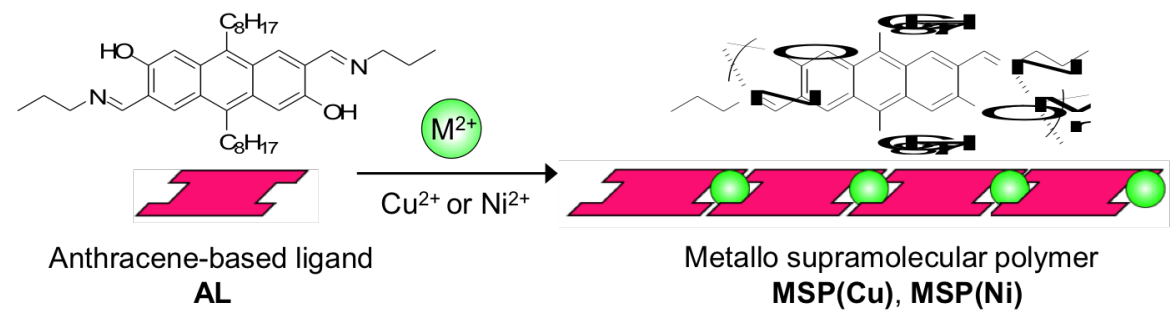

b

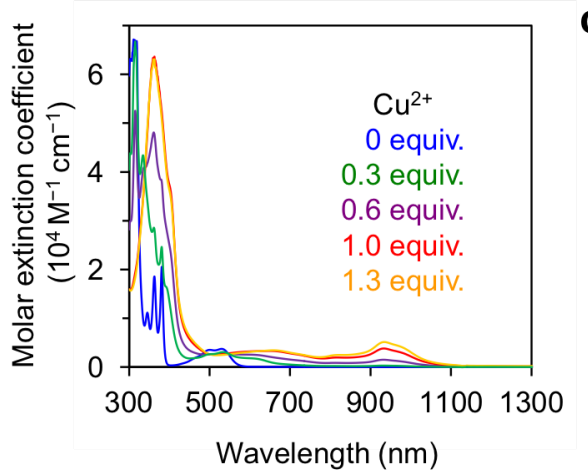

C

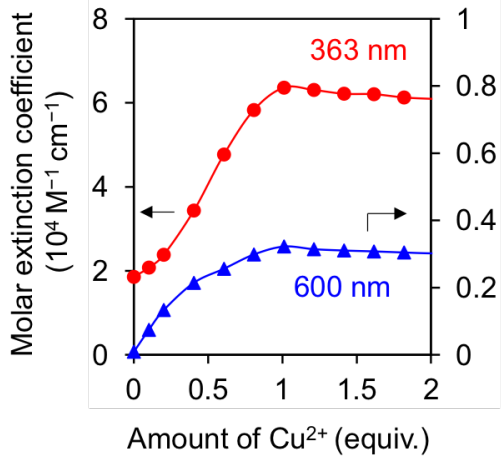

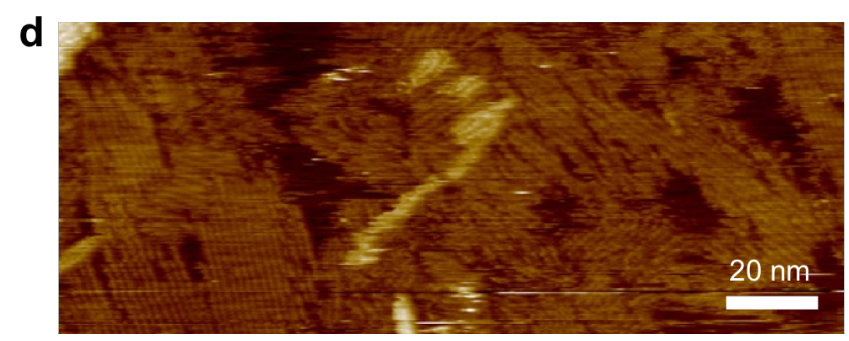

Figure 1. Formation of metallo-supramolecular polymers (MSPs). (a) Chemical structures of AL and MSP. (b),(c) UV-Vis titration experiments of $\mathrm{AL}\left(7.0 \times 10^{-5} \mathrm{M}, o\right.$-DCB/methanol $\left.=9 / 1\right)$ with $\mathrm{Cu}(\mathrm{AcO})_{2} \cdot \mathrm{H}_{2} \mathrm{O}(10.6 \times \mathrm{mM}$, methanol $)$ at room temperature. The optical path length was $10 \mathrm{~mm}$. (d) STM image of MSP(Cu) assembled on highly-oriented pyrolytic graphite (HOPG).

\section{RESULTS AND DISCUSSION}

Formation of MSP. Polarizable polycyclic aromatic molecules exhibit strong van der Waals interactions with SWCNTs, and as a result we have chosen anthracene-based ligands (AL) shown in Fig. 1 a to produce dispersions. The anthracene core is substituted with two $n$-propyl salicylaldimine motifs, which are expected to form a square planar complex with $\mathrm{Cu}^{2+}$ or $\mathrm{Ni}^{2+}$ ions. ${ }^{16,17}$ Two $n$-octyl chains were added to the 9,10-positions of the anthracene to solubilize the SWCNT dispersion. The ability of this ligand to be converted into a ladder polymer with transition metal linkages was studied by titration with $\mathrm{Cu}^{2+}$ or $\mathrm{Ni}^{2+}$ ions. The UV-Vis absorption of $\mathrm{AL}$ varied upon addition of $\mathrm{Cu}^{2+}$ and abruptly saturated with one equivalent of $\mathrm{Cu}^{2+}$ (Fig. 1b, 1c). This result indicates that salicylaldimine units strongly coordinate $\mathrm{Cu}^{2+}$, yielding $\operatorname{MSP}(\mathrm{Cu})$. In contrast, the $\mathrm{Ni}^{2+}$ titration curve with AL lacked a clear saturation point (Fig. S1), suggesting that MSP(Ni) is oligomeric in solution. Gel permeation chromatography experiment reveals that $\mathrm{MSP}(\mathrm{Cu})$ displays a radius of gyration that is comparable to that of $\pi$-conjugated polymer with $\mathrm{M}_{\mathrm{n}}=9.0 \mathrm{k}$, thereby confirming that $\operatorname{MSP}(\mathrm{Cu})$ is approximately $15-$ mers on average (Fig. S2). The polymeric structure of $\operatorname{MSP}(\mathrm{Cu})$ was also confirmed by assembling it on a graphite surface and imaging by scanning tunneling microscope (STM). As shown in Fig. 1d, these studies revealed linear and rod-like assemblies of MSP $(\mathrm{Cu})$ with domain size of $10-50 \mathrm{~nm}$, corresponding to $10-50$ mers.
Wrapping SWCNT with MSP. A key element of our design is that the monomers are not sufficient to disperse SWCNTs, but that the collective properties of the supramolecular polymer create a highly stable dispersion. ${ }^{18,19} \mathrm{AL}$ meets these criteria and is not capable of forming stable SWCNT dispersions in $o$-dichlorobenzene (o-DCB), however with sonication $(5 \mathrm{~min})$ in the presence of $\mathrm{Cu}^{2+}$ or $\mathrm{Ni}^{2+}$ ions homogeneous stable SWCNT dispersions are created (Fig. 2a). In contrast, similar procedures complexing AL with $\mathrm{Zn}^{2+}$ and $\mathrm{Co}^{2+}$ did not produce stable SWCNT dispersions, presumably due to the lack of square-planar configuration about the metal ion (Fig. S3). To determine if small molecule square planar metal complexes are sufficient, we investigated the $\mathrm{Ni}^{2+}$ complex of $n$ dodecylsalicylaldimine (DSA(Ni)), which has an established square planar structure, ${ }^{17}$ and find that this monomeric system is incapable of producing stable SWCNT dispersions (Fig. S4). As a result, it would appear that the MSP structure having square planar metal center, polarizable $\pi$ electron system, and polymeric structure are collectively responsible for producing well behaved SWCNT dispersions. The quality of the dispersions is further confirmed by UV-Vis-NIR spectra of solutions of SWCNT/MSP $(\mathrm{Cu})$ and SWCNT/MSP(Ni) that revealed well-resolved absorptions from $\mathrm{E}_{11}$ transitions of SWCNT, which is indicative of isolated (debundled) SWCNTs (Fig. 2b). ${ }^{20}$ Scanning electron microscopy (SEM) images of SWCNT/MSP $(\mathrm{Cu})$ drop-cast film revealed SWCNTs having more linear and narrow structures relative to pristine SWCNTs, also supporting the formation of isolated wrapped 


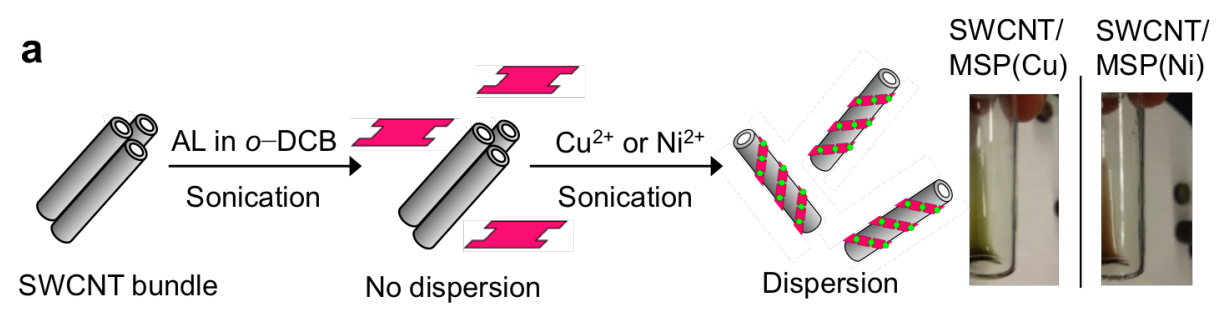

b
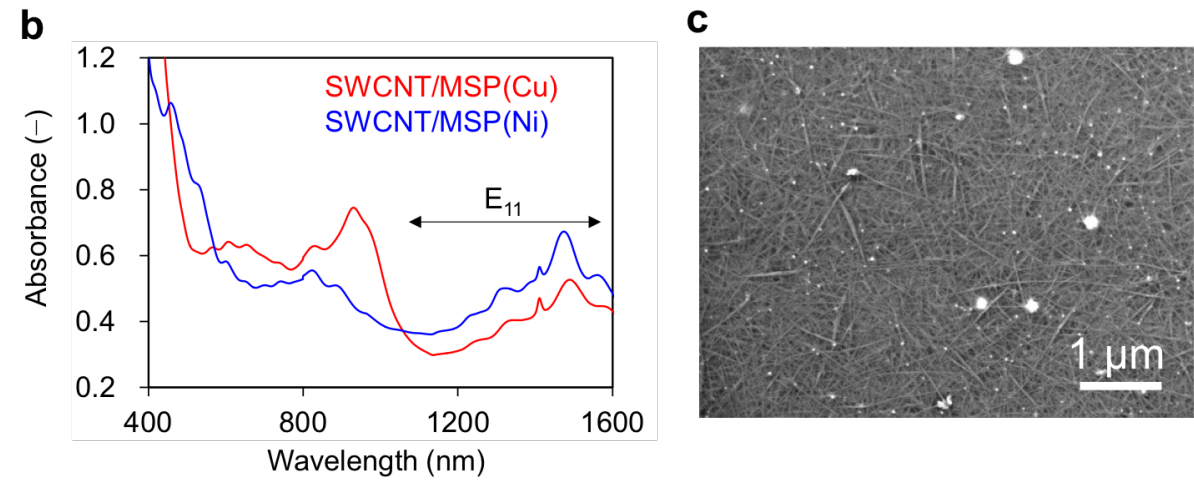

Figure 2. Dispersion of SWCNT with MSPs. (a) Scheme for dispersing SWCNT with MSPs. Equal equivalents of $\mathrm{Cu}(\mathrm{AcO})_{2} \cdot \mathrm{H}_{2} \mathrm{O}$ or $\mathrm{Ni}(\mathrm{AcO})_{2} \cdot 4 \mathrm{H}_{2} \mathrm{O}$ in methanol $(10.6 \mathrm{mM}, 82 \mu \mathrm{L})$ and $\mathrm{AL}(0.5 \mathrm{mg})$ in $o$-DCB $(1 \mathrm{~mL})$ are combined with SWCNTs $(0.1 \mathrm{mg})$, and then sonicated for $5 \mathrm{~min}$. (b) UV-Vis-NIR spectra of SWCNT dispersed by $\mathrm{MSP}(\mathrm{Cu})$ and $\mathrm{MSP}(\mathrm{Ni})$ in $o-\mathrm{DCB}$ (optical pathlength: $1 \mathrm{~mm})$. (c) SEM image of a drop-cast film of SWCNT/MSP $(\mathrm{Cu})$ on Si substrate. The concentration of SWCNT $(0.1 \mathrm{mg} / \mathrm{mL})$ was identical to that used to create networks for the sensing studies.

SWCNTs (Fig. 2c, Fig. S5). The assembly of isolated SWCNTs into random porous network structures facilitates the diffusion of analyte molecules into the film and the triggered generation of enhanced electronic conduction pathways via disassembly of the polymer wrappers.

Sensing properties of SWCNT/MSP. Chemiresistive sensors were prepared by drop-casting $0.5 \mu \mathrm{L}$ of $\operatorname{SWCNT} / \mathrm{MSP}(\mathrm{Cu})$ or SWCNT/MSP(Ni) dispersions on interdigitated gold electrodes ( $0.2 \mathrm{~mm}$ gaps), and the variation in conductivity upon exposure to various types of analyte vapors, including DECP, is measured by detecting the current with an applied potential of $0.1 \mathrm{~V}$ (Fig. 3a). Only very small amounts of material are required to make a sensor and up to 4,000 sensors can be prepared from $1 \mathrm{mg}$ of AL. The quality of the SWCNT dispersion enables high sensor fabrication reproducibility from batch-to-batch (Table S1). ${ }^{21}$ The SWCNT/MSP composites proved effective in generating high sensitivity to target analytes as shown in Fig. 3b. Specifically, SWCNT/MSP $(\mathrm{Cu})$ and SWCNT/MSP(Ni) display large increases in conductivity upon exposure to $10 \mathrm{ppm}$ DECP when compared to the responses of sensors created with pristine-SWCNTs and SWCNTs wrapped by poly(9,9-di- $n$-octylfluorenyl-2,7-diyl) (PFO), which were prepared under the same conditions. PFO stabilized SWCNT dispersions have been studied previously ${ }^{22}$ were chosen as a non-responsive control material. It is also noteworthy that SWCNT/MSP exhibits enhanced sensitivity to specific chemicals such as DECP, while responses to water as well as a number of common volatile organic compounds (VOCs) are much lower and similar what is observed for the pristine-SWCNT and SWCNT/PFO controls (Fig. 3c). Moreover, in most cases, the response to non-target analytes is an increase in resistivity, consistent with swelling- or hole-quenching type transduction mechanism. ${ }^{8}$ This is opposite that observed for DECP wherein the disassembly of the MSP wrapper generates lower resistance junctions between the SWCNTs.
Optimization of SWCNT/MSP(Cu) sensor for enhanced response. The SWCNT/MSP $(\mathrm{Cu})$ composition was optimized by monitoring the chemiresistive response to concentrated dimethylchlorophosphate (DMCP) vapor in dry argon (Ar). The 1:5 (by weight) mixture of SWCNT to $\operatorname{MSP}(\mathrm{Cu})$ displayed a higher response than 1:1, 1:2, and 1:10 mixtures (Fig. S6). It is also found that the use of 0.5 eq. of $\mathrm{Cu}^{2+}$ lead to lower response (Fig. S7), suggesting that complete polymerization of MSP is important to achieve the maximum response. The change of solvent from $o$ $\mathrm{DCB}$ to a mixture of $o$-DCB and toluene (1:4 by volume) led to a twofold increase of the response to DMCP, and a further twofold increase was achieved when the supernatant of centrifuged solution was used (Fig. S8). The drop-cast film of the SWCNT/MSP $(\mathrm{Cu})$ supernatant $o$-DCB/toluene solution gave sensors with higher initial resistance (ca. $2 \mathrm{M} \Omega$ for $5 \mu \mathrm{L} \times 3$ drops) than those made from the as formed dispersion (ca. $100 \mathrm{k} \Omega$ for $5 \mu \mathrm{L} \times 3$ drops ), suggesting that a minority amount of bundled SWCNTs that are removed by centerfugation are sufficient to cause low resistance pathways that decrease the sensitivity. In addition, when compared with SWCNT/MSP $(\mathrm{Cu})$ dispersed by $o$-DCB, the UV-Vis-NIR spectrum of the supernatant solution revealed reduced signal from $\mathrm{MSP}(\mathrm{Cu})$ chromophore appearing at $750 \mathrm{~nm}$ and $940 \mathrm{~nm}$ (Fig. S9). These results suggest that toluene (a poor solvent for $\operatorname{MSP}(\mathrm{Cu})$ ) serves to remove excess $\mathrm{MSP}(\mathrm{Cu})$. Surplus polymer that impedes optimal SWCNT-SWCNT contacts with the analyte triggered unwrapping of $\operatorname{MSP}(\mathrm{Cu})$ is also likely to be detrimental to the sensor sensitivity. The addition of toluene doesn't effect the $\mathrm{E}_{11}$ transitions of SWCNT, indicating that the SWCNT composition (i.e., chirality or diameter) is not effected by the change in solvent. ${ }^{23}$ As shown in Fig. $4 \mathrm{a}$, the optimized SWCNT/MSP $(\mathrm{Cu})$ sensor demonstrated a $2,000 \%$ increase in conductivity upon exposure to $10 \mathrm{ppm}$ DECP. Accordingly, optimization improved the response by $6 \mathrm{X}$, and is 40 and 200 times larger than that of pristine SWCNT and SWCNT/PFO controls, respectively (in comparison with Fig. $3 b$ ). 
a

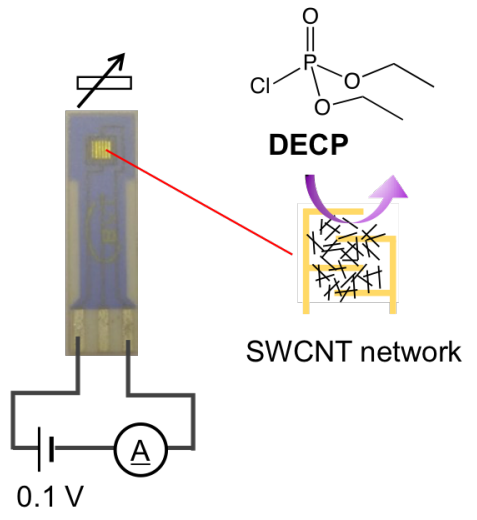

b

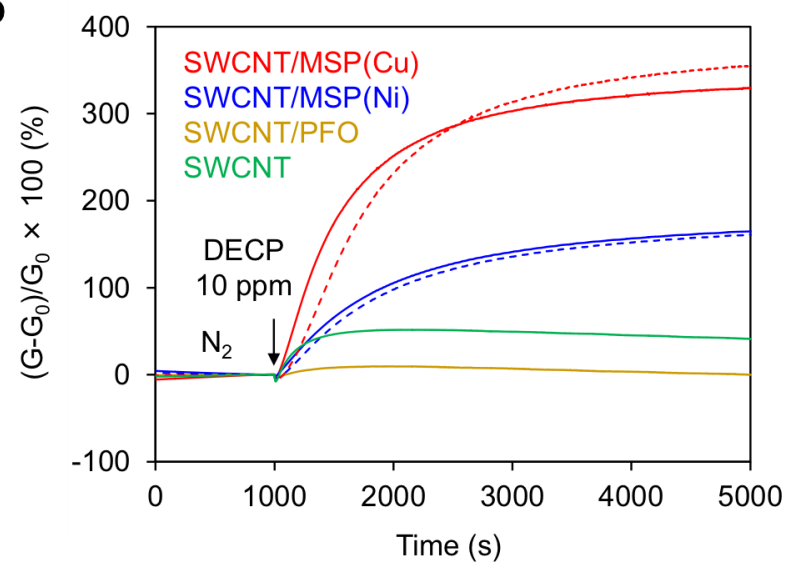

C

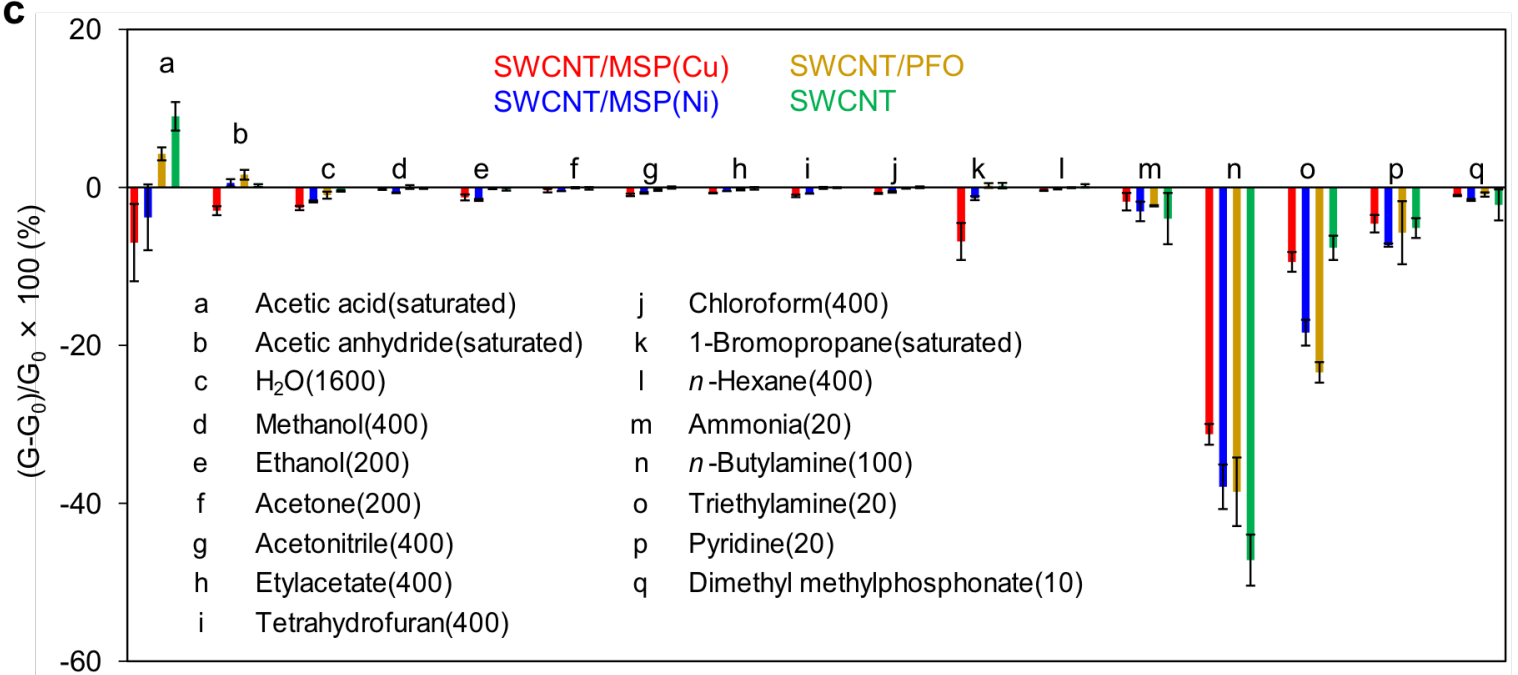

Figure 3. Chemical sensing properties of SWCNT wrapped with MSP. (a) Configuration of SWCNT-based chemiresistive sensor. (b) Conductance traces of SWCNT-based chemiresistive sensors upon exposure to $10 \mathrm{ppm}$ DECP in $\mathrm{N}_{2}$. Two samples of SWCNT/MSP were tested for confirming reproducibility (shown by solid and dotted line). Initial resistance of sensors were $15 \mathrm{k} \Omega-30 \mathrm{k} \Omega$. (c) Chemiresisitve responses of SWCNT-based chemiresistive sensors upon $50 \mathrm{~s}$ exposure to various vapors in $\mathrm{N}_{2}$ (concentration in ppm given in parentheses). Chemiresisitve responses to these analytes were nearly saturated within $50 \mathrm{~s}$.

The saturated chemiresistive responses for $1.0 \mathrm{ppm}$ and $0.1 \mathrm{ppm}$ DECP were ca. $600-700 \%$, and the slope (rate) of increasing conductivity decreased as the concentration of DECP decrease.

Investigating the sensing mechanism. As expected, SWCNT/MSP $(\mathrm{Cu})$ as well as $\mathrm{SWCNT} / \mathrm{MSP}(\mathrm{Ni})$ displays increases in conductivity upon exposure to other strong electrophiles such as acetyl chloride, trifluoroacetic acid, and $\mathrm{SOCl}_{2}$ (Fig. S3, S10). To investigate the sensing mechanism, diamagnetic square planar $\mathrm{Ni}^{2+}$ complex of $n$-dodecylsalicylaldimine (DSA(Ni)) was used as a model compound (Fig. S11). ${ }^{17}$ After bubbling with DMCP for 3 min the ${ }^{1} \mathrm{H}-\mathrm{NMR}$ spectrum indicates that the DSA(Ni) is completely demetallated and only dissociated free DSA is detected. The formation of an iminium ion $^{24}$ is a likely intermediate step of demetallation; however such a highly reactive intermediate will not persist to allow detection. When acetyl chloride was added to DSA(Ni), free DSA as well as the DSA acetyl-ester were observed in ${ }^{1} \mathrm{H}-\mathrm{NMR}$ spectrum (Fig. S11). This result indicates that there may be multiple reaction pathways for demetallation of MSP and/or post demetallation chemical transformations. UV-Vis-NIR and MALDI-TOF MS experiments also support that demetallation of $\mathrm{MSP}(\mathrm{Cu})$ occurs with exposure to DMCP (Fig. S12).

Fragments of MSP (including AL itself) are not effective at wrapping or dispersing SWCNTs; bubbling DMCP vapor through a SWCNT/MSP $(\mathrm{Cu})$ dispersion in $o$-DCB gives immediate precipitation (Fig. S13). In contrast, SWCNTs dispersed using PFO do not precipitate even after several minutes of bubbling DMCP vapor into the solution. PFO does not react with DMCP and it is clear that the chemical disassembly of the MSP is a key component of this system's response to strong electrophiles.

Modification of AL with oxime for improved response to DECP. In order to further increase the transitional response (i.e., slope of increasing conductivity) to DECP, $n$-propyl imines in AL were substituted by oximes to form ALOx (Fig. 4b). Fast and irreversible reactivity of oximes with phosphorous electrophiles, which is explained as by the a-effect, has been investigated extensively, and underlies the structure-function basis for nerve agent antidotes (e.g., pralidoxime shown in Fig. 4b). ${ }^{25}$ Although ALOx forms a strong metal ligand complex with $\mathrm{Cu}^{2+}$ in 1:1 molar stoichiometry (Fig. S14), MSP $(\mathrm{Cu})$ made from ALOx was not capable 

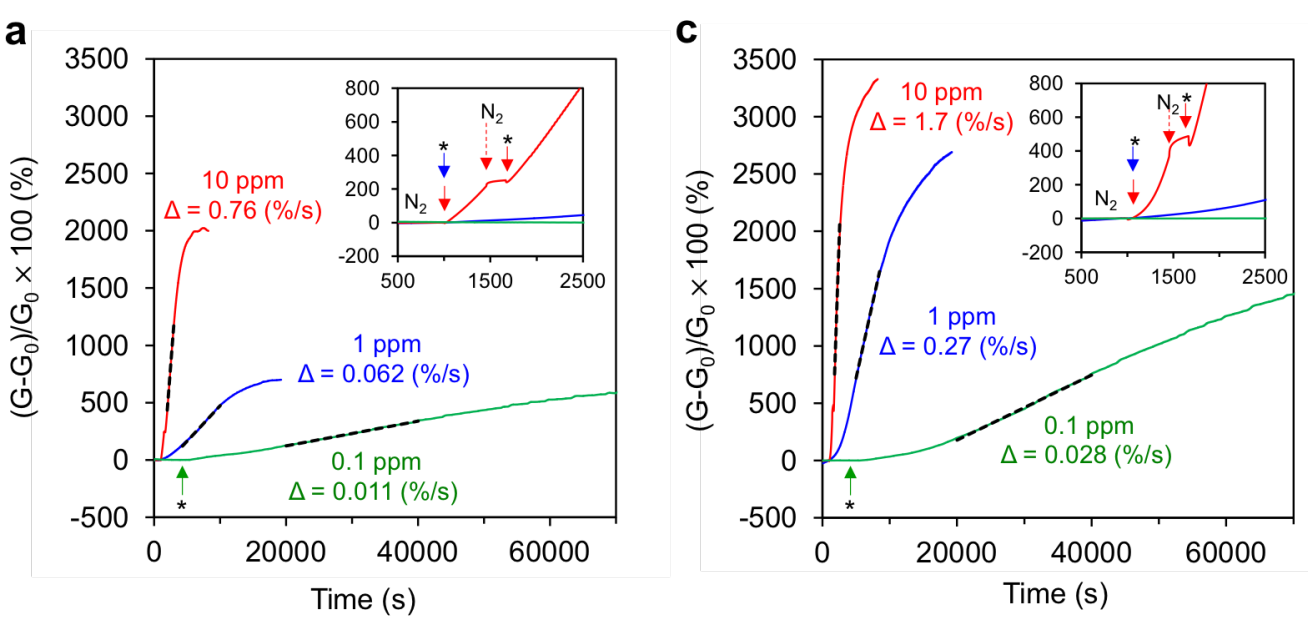

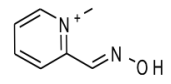

Pralidoxime

(Antidote for nerve agent)

Figure 4. Monitoring of trace DECP using optimized $\operatorname{SWCNT} / \mathrm{MSP}(\mathrm{Cu})$ sensors. (a) Conductance traces of optimized SWCNT/MSP $(\mathrm{Cu})$ chemiresistive sensors upon exposure to 0.1, 1.0, and $10 \mathrm{ppm} \mathrm{DECP} \mathrm{in} \mathrm{N}_{2}$. A mixture of $o$-DCB and toluene (1:4 by volume) was used for dispersing SWCNT/MSP(Cu), and the supernatant of the centrifuged solution was drop-cast on the electrode to prepare sensors. The initial resistance range of the sensors was $700 \mathrm{k} \Omega-2,000 \mathrm{k} \Omega$. Arrows indicate when injection of gas started. Asterisk denotes DECP. Black dotted lines indicate the slope of the traces estimated by linear approximation. Baseline correction was applied to the $0.1 \mathrm{ppm}$ sensing trace (see Fig. S15). (b) Chemical structures of ALOx and pralidoxime. (c) Conductance traces of optimized SWCNT/MSP $\left(\mathrm{Cu}\right.$ ) chemiresistive sensors made from 0.5:0.5:1.0 (by mole) mixtures of ALOx, $\mathrm{AL}$, and $\mathrm{Cu}^{2+}$ upon exposure to $0.1,1.0$, and $10 \mathrm{ppm}$ DECP in $\mathrm{N}_{2}$. A mixture of $o-\mathrm{DCB}$ and toluene (1:4 by volume) was used for dispersing SWCNT/MSP $(\mathrm{Cu})$, and the supernatant of the centrifuged solution was drop-cast on the electrode for preparing sensors. The initial resistance range of sensors was $700-2,000 \mathrm{k} \Omega$.

of dispersing SWCNTs. Thus, composite $\mathrm{MSP}(\mathrm{Cu})$ made from 0.5:0.5:1.0 mixture of ALOx, $\mathrm{AL}$, and $\mathrm{Cu}^{2+}$ (by mole) was utilized to disperse SWCNTs in a solution of $o$-DCB and toluene (1:4 by volume). Chemiresistive sensors were prepared from the supernatant of the centrifuged mixture, and Fig. 4c shows that the slope of increasing conductivity as well as saturated response are significantly improved by incorporating ALOx into the sensor formulation. The slope of chemiresistive response to DECP decreases with decreasing DECP concentration, while the response saturates at a similar level. This result indicates a nearly ideal dosimetric sensing response that is directly correlated with the number of analyte (DECP) molecules that MSP encounters.

This dosimetric behavior is consistent with a mechanism wherein the SWCNT/MSP network is initially in a highly resistive form, wherein the MSP groups are preventing optimal charge transport across SWCNT-SWCNT junctions. DECP triggered disassembly of the MSP wrapper enhances the conductivity and at lower levels of disassembly new independent pathways are being established with each improved SWCNT-SWCNT junction. As the MSP is progressively disassembled, new bifurcated and redundant pathways have less of an overall effect and the relative response decreases. This sensing mechanism is dosimetric giving a time- and concentration-integrated response. As indicated previously, dosimeters are particularly useful in monitoring acutely toxic chemical substances at extremely low concentrations. ${ }^{6,26}$ Although our present investigations are limited to chemiresistors, we anticipate that additional sensitivity enhancements may be possible if our sensing materials were to be integrated into devices that function in capacitive or field effect transistor modes. ${ }^{5,6}$

Wireless chemical sensing. The utility of the large and irreversible chemiresistive response of SWCNT/MSP $(\mathrm{Cu})$ sensors was demonstrated by wireless chemical sensing under ambient conditions. To this end, a near-field communication (NFC) $\operatorname{tag}^{27}$ was modified such that a commercial smartphone can be used to detect trace toxic chemicals. Using a Samsung Galaxy S4 (SGS4), we find that a turn-on dosimetric sensor can be prepared by inserting resistors in series with the antenna ( $L)$ of NFC tag (Fig. 5a). If the inserted resistance is higher than $2.2 \mathrm{k} \Omega$, the modified NFC tag was unreadable by SGS4 because resistance is high enough to prohibit the NFC tag from creating a resonant circuit at $13.56 \mathrm{MHz}$ (Fig. S16). In contrast, the modified NFC tag becomes readable by SGS4 when the inserted resistance is lower than $1.0 \mathrm{k} \Omega$. Thus, approximately $100 \%$ increase in conductivity (i.e., change from $2.2 \mathrm{k} \Omega$ to $1.0 \mathrm{k} \Omega$ ) is required to switch between the unreadable and readable state, and hence our chemiresistive dosimetric sensor is well suited to creating a turn-on NFC tag (Fig. 4a, c). As a demonstration, an NFC tag modified by our SWCNT/MSP(Cu) material (initial resistance $=3.6 \mathrm{k} \Omega$ ) is initially unreadable by SGS4, but converts to a readable state $($ resistance $=0.79 \mathrm{k} \Omega$ ) after $5 \mathrm{~s}$ exposure to $10 \mathrm{ppm}$ $\mathrm{SOCl}_{2}$ in air (Fig. 5c). The NFC tag remains readable by SGS4 after more than 1 week of storage in ambient atmosphere, and hence the cumulative $\mathrm{SOCl}_{2}$ exposure history can be established even after an extended period of time. This non-volatile memory is advantageous for wireless sensing especially when continuous monitoring 

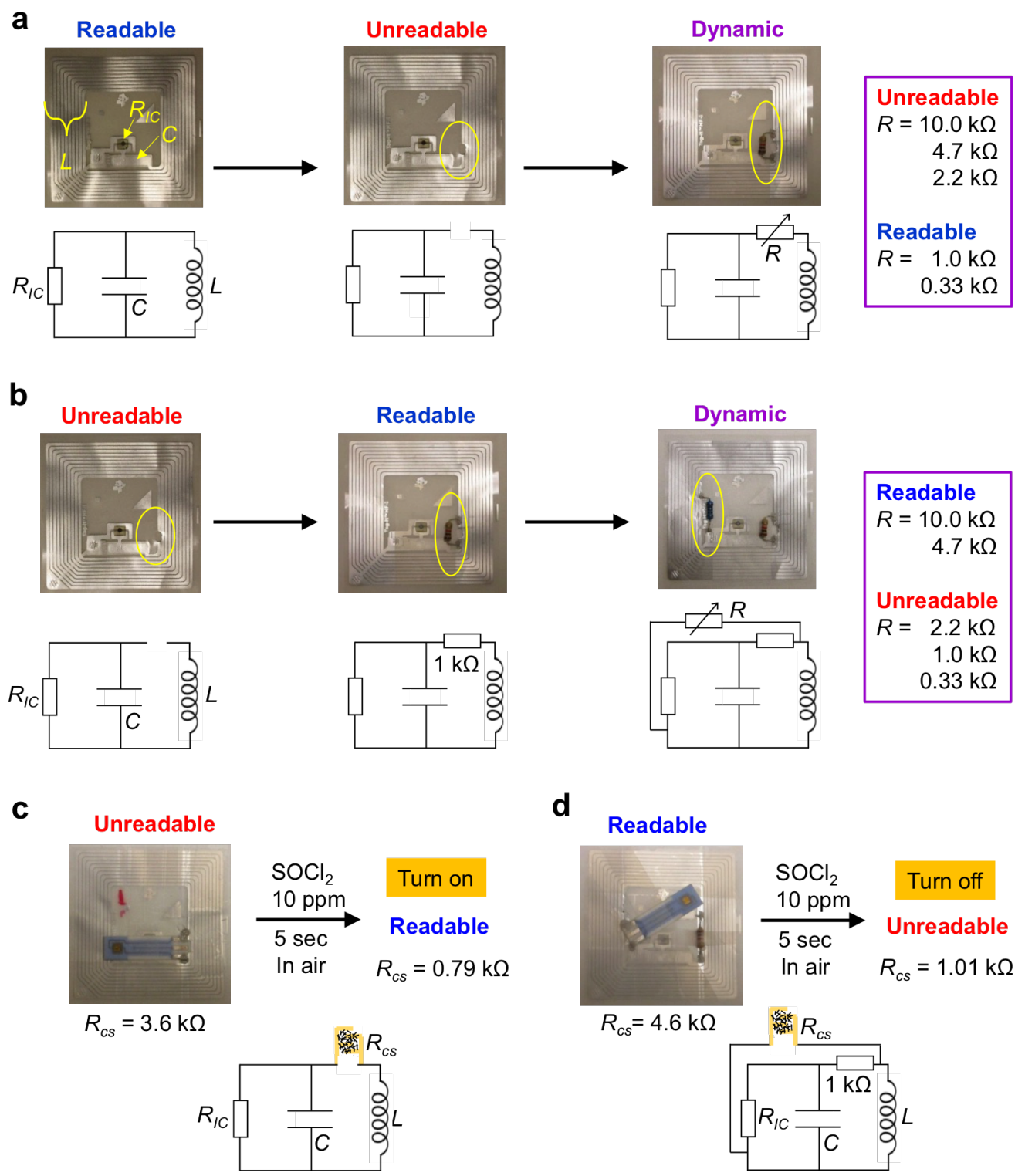

Figure 5. Modification of NFC tag and smart phone detection of thionyl chloride in air. (a) Modification of NFC tag for circuit design of turn-on sensor. $R_{I C}, C, L$, and $R$ denote the integrated circuit, capacitor, antenna, and resistor, respectively. (b) Modification of NFC tag for circuit design of turn-off sensor. (c) Wireless $\mathrm{SOCl}_{2}$ sensing using smart phone and NFC tag modified with SWCNT/MSP(Cu) chemiresistive sensor $\left(R_{c s}\right)$. The modified NFC tag becomes readable by a smart phone after $5 \mathrm{~s}$ exposure to $10 \mathrm{ppm} \mathrm{SOCl}_{2}$ at ambient condition (in air, $17.1^{\circ} \mathrm{C}$, relative humidity $=16.2 \%$ ). A mixture of $o$-DCB and toluene $(1: 1$ by volume) was used for dispersing SWCNT/MSP $(\mathrm{Cu})$, and the supernatant of the centrifuged solution was used for preparing sensors. (d) Wireless $\mathrm{SOCl}_{2}$ sensing using a smart phone and NFC tag modified with SWCNT/MSP $(\mathrm{Cu})$ chemiresistive sensor $\left(R_{c s}\right)$. The modified NFC tag becomes unreadable by a smart phone after $5 \mathrm{~s}$ exposure to $10 \mathrm{ppm} \mathrm{SOCl}_{2}$.

is economically unfeasible or difficult as a result of limited sampling opportunities. We expect that this specific sensing system could find utility for leak detection from $\mathrm{Li}-\mathrm{SOCl}_{2}$ backup batteries commonly used in medical instruments, fire alarms, and military systems. ${ }^{28}$

An alternative turn-off dosimetric sensor can be prepared by changing the circuit design (Fig. $5 \mathrm{~b}$ ). In this case a $1 \mathrm{k} \Omega$ resistor is inserted in series with the antenna $(L)$ of NFC tag and the variable resistor (chemiresistive dosimeter) is then inserted as shown in Fig. $5 \mathrm{~b}$. The variable resistor is competitive with the added $1 \mathrm{k} \Omega$ resistor, and the resulting irregular conductive pathway disturbs the resonant properties of NFC tag only when the resistance through the added sensory material is low. Experimentally, an NFC tag modified with a $4.7 \mathrm{k} \Omega$ variable resistor was readable by smart phone, while an NFC tag with $2.2 \mathrm{k} \Omega$ variable resistor was unreadable (Fig. S17). This concept is realized by inserting SWCNT/MSP $(\mathrm{Cu})$ sensor with initial resistance $=4.6 \mathrm{k} \Omega$ to the NFC tag (Fig. $5 \mathrm{~d}$ ). The modified NFC tag was initially readable by smart phone, and became unreadable after $5 \mathrm{~s}$ exposure to $10 \mathrm{ppm} \mathrm{SOCl}_{2}$ in air. The combination of turn-on and turn-off sensor modes can enhance the reliability of a sensing system. These simple modification techniques to conventional NFC tags facilitate deployment of our methods as chemical sensors for safety and security management. 


\section{CONCLUSION}

In conclusion, SWCNT wrapped with MSPs display large timeand concentration-integrated chemiresistive responses as a result of a triggered disassembly of the MSPs induced by strong electrophiles such as DECP and $\mathrm{SOCl}_{2}$. Various approaches including ligand design, selection of metal ions, and optimizing SWCNT dispersion conditions are demonstrated to be effective to increase sensitivity to harmful analytes. Demetallation (disassembly) of the MSP degrades the SWCNT wrapper, thereby decreasing interSWCNT resistance at junctions, resulting in large $(>1,000 \%)$ increases in conductivity. The highly sensitive and irreversible chemiresistive chemical responses enable wireless chemical sensing with NFC technology. Our findings reveal that wrapping of SWCNTs with chemically-degradable polymers is a powerful strategy for the development of advanced chemiresistive dosimetric sensors. The choice of the supramolecular polymer is crucial to these designs and the polymer must generate stable dispersions that can be used to create solid composites that prevent low resistivity inter-SWCNT contacts. After disassembly, the molecules must release the SWCNTs and not quench their conductivity to generate low resistivity networks with low resistivity inter-SWCNT junctions. We expect to create additional families of highly sensitive chemiresistive dosimeters based on this concept.

\section{ASSOCIATED CONTENT}

Supporting Information. General experimental procedures, sensing experimental procedures, and synthesis and characterization of ligand molecules. This material is available free of charge via the Internet at http://pubs.acs.org.

\section{AUTHOR INFORMATION}

\section{Corresponding Author}

*tswager@mit.edu

\section{Notes}

The authors declare no competing financial interest.

\section{ACKNOWLEDGMENT}

This work was supported by the National Science Foundation DMR-1410718 and a JSPS Fellowship for Research Abroad (to S.I.). Bora Yoon (MIT) is acknowledged for SEM measurements. Marshall H. Craft (MIT) is acknowledged for the development of a network analyzer signal processing LabVIEW program. $\mathrm{Li} \mathrm{Li}$ (MIT) is acknowledged for mass analysis.

\section{REFERENCES}

(1) Taylor, R. F.; Schultz, J. S. Handbook of Chemical and Biological Sensors (IOP Publishing, 1996).

(2) Korotcenkov, G. Handbook of Gas Sensor Materials: Properties, Advantages and Shortcomings for Applications Volume 2: New Trends and Technologies (Springer, 2007).

(3) Neri, G. Chemosensors 2015, 3, 1-20.

(4) Campbell, M. G.; Sheberla, D.; Liu, S. F.; Swager, T. M.; Dincă, M. Angew. Chem. Int. Ed. 2015, 54, 4349-4352.
(5) Kauffman, D. R.; Star, A. Angew. Chem. Int. Ed. 2008, 47, 6550-6570.

(6) Snow, E. S.; Perkins, F. K.; Robinson, J. A. Chem. Soc. Rev. 2006, 35, 790-798.

(7) Schnorr, J. M.; Swager, T. M. Chem. Mater. 2011, 23, 646-657.

(8) Fennell, J. F., Jr.; Liu, S. F.; Azzarelli, J. M.; Weis, J. G.; Rochat, S.; Mirica, K. A.; Ravnsbæk, J. B.; Swager, T. M. Angew. Chem. Int. Ed. 2016, 55, 1266-1281.

(9) Fujigaya, T.; Nakashima, N. Sci. Tech. Adv. Mater. 2015, 16, 024802 .

(10) Romano, J. A., Jr.; Lukey, B. J.; Salem, H. Chemical Warfare Agents: Chemistry, Pharmacology, Toxicology, and Therapeutics, Second Edition (CRC Press, 2007).

(11) Lobez, J. M.; Swager, T. M. Angew. Chem. Int. Ed. 2010, 49, 95-98.

(12) Aida, T.; Meijer, E. W.; Stupp, S. I. Science 2012, 335, 813-817.

(13) Wojtecki, R. J.; Meador, M. A.; Rowan, S. J. Nat. Mater. 2011, 10, 14-27.

(14) Whittell, G. R.; Hager, M. D.; Schubert, U. S.; Manners, I. Nat. Mater. 2011, 10, 176-188.

(15) Yang, L.; Tan, X.; Wang, Z.; Zhang, X. Chem. Rev. 2015, 115, 7196-7239.

(16) Sacooni, L.; Ciampolini, M. J. Chem. Soc. 1964, 276-280.

(17) Chakravorty, A.; Fennessey, J. P.; Holm, R. H. Inorg. Chem.1965, 4, 26-33.

(18) Toshimitsu, F.; Nakashima, N. Nat. Commun. 2014, 5, 5041.

(19) Llanes-Pallas, A.; Yoosaf, K.; Traboulsi, H.; Mohanraj, J.; Seldrum, T.; Dumont, J.; Minoia, A.; Lazzaroni, R.; Armaroli, N.; Bonifazi, D. J. Am. Chem. Soc. 2011, 133, 15412-15424.

(20) Samanta, S. K.; Fritsch, M.; Scherf, U.; Gomulya, W.; Bisri, S. Z.; Loi, M. A. Acc. Chem. Res. 2014, 47, 2446-2456.

(21) Zhang, Y.; Xu, M.; Bunes, B. R.; Wu, N.; Gross, D. E.; Moore, J. S.; Zang, L. ACS Appl. Mater. Interfaces 2015, 7, 7471-7475.

(22) Nish, A.; Hwang, J.-Y.; Doig, J.; Nicholas, R. J. Nat. Nanotech. 2007, 2, 640-646.

(23) Hwang, J.-Y.; Nish, A.; Doig, J.; Douven, S.; Chen, C.-W.; Chen, L.-C.; Nicholas, R. J. J. Am. Chem. Soc. 2008, 130, 3543-3553.

(24) Weinreb, S. M.; Scola, P. M. Chem. Rev. 1989, 89, $1525-1534$.

(25) Dale, T. J.; Rebek, J., Jr. Angew. Chem. Int. Ed. 2009, 48, 7850-7852.

(26) Mioduszewski, R.; Manthei, J.; Way, R.; Burnett, D.; Gaviola, B.; Muse, W.; Thomson, S.; Sommerville, D.; Crosier, R. Toxicol. Sci. 2002, 66, 176-184.

(27) Azzarelli, J. M.; Mirica, K. A.; Ravnsbæk, J. B.; Swager, T. M. Proc. Natl. Acad. Sci. USA 2014, 111, 18162-18166.

(28) http://www.powertechsystems.eu/home/products/primarycells-lithium-thionyl-chloride-li-soc12/ (Accessed on April 13, 2016). 
1

2

3

4

5

6

7

8

9

10

11

12

13

14

15

16

17

18

19

20

21

22

23

24

25

26

27

28

29

30

31

32

33

34

35

36

37

38

39

40

41

42

43

44

45

46

47

48

49

50

51

52

53

54

55

56

57

58

59

60

Table of Contents artwork

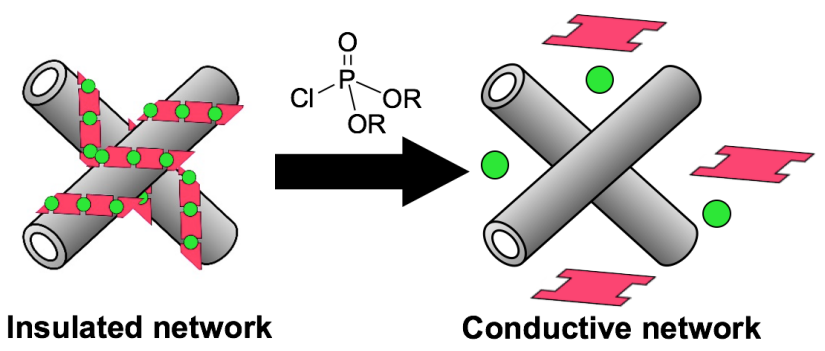

ve network 